\title{
Acanthamoeba T4 and T15 genotypes associated with keratitis infections in Italy
}

\author{
D. Di Cave • R. Monno • P. Bottalico • S. Guerriero • \\ S. D'Amelio • C. D'Orazi • F. Berrilli
}

Received: 18 September 2008 / Accepted: 29 November 2008/Published online: 18 December 2008

(C) Springer-Verlag 2008

\begin{abstract}
Thus far there is little data available concerning Acanthamoeba associated amoebic keratitis (AK) from Italy. In order to understand the incidence of Acanthamoeba in patients with ocular infections and to characterize the isolates at the molecular level, ocular specimens and contact lenses or lens case solutions from 140 patients were analysed by culture and by an 18S rRNA (Rns) genebased PCR method. Nineteen (13.6\%) patients showed Acanthamoeba culture positive samples. Eleven out of the 14 genetically characterized isolates were assigned to the
\end{abstract}

\footnotetext{
D. Di Cave $\cdot$ F. Berrilli $(\bowtie)$

Department of Public Health and Cell Biology,

University of Rome Tor Vergata,

Via Montpellier 1,

Rome, Italy

e-mail: berrilli@uniroma2.it

D. Di Cave $\cdot$ C. D’Orazi

Azienda Ospedaliera Universitaria Policlinico Tor Vergata,

Viale Oxford 81,

Rome, Italy

R. Monno $\cdot$ P. Bottalico

Department of Internal Medicine and Public Health Hygiene

Section, University of Bari,

P. G. Cesare 11,

Bari, Italy

S. Guerriero

Department of Ophthalmology and Otorhinolaryngology,

University of Bari,

P. G. Cesare 11,

Bari, Italy

\section{S. D'Amelio}

Department of Public Health Sciences,

Sapienza University of Rome,

P.le Aldo Moro 5,

Rome, Italy
}

T4 genotype. Three isolates, two of them from patients with keratitis responding to specific anti-Acanthamoeba therapy, were identified as belonging to the T15 genotype. This finding represents the first association between the T15 genotype and human amoebic keratitis. PCR amplification of the 18S ribosomal DNA proved to be a sensitive method, potentially able to detect Acanthamoeba without the need of long culture incubation, and thus considerably useful for clinical applications.

\section{Introduction}

Acanthamoeba spp. are ubiquitous free-living amoebae, typically widespread in soil and water and known as opportunistic protozoa able to infect humans. In healthy individuals, Acanthamoeba can be a cause of infections of the central nervous system, as well as of amoebic keratitis (AK), a seriously painful and vision-threatening ulceration of the cornea [1].

The taxonomy of the genus is mainly based on morphological features such as size and cyst morphology [2], and only recently the application of molecular tools, in particular sequencing of $18 \mathrm{~S}$ rRNA genes, has allowed the classification of Acanthamoeba into 15 genotype groups designated as T1-T15 [3-7]. Amoebic keratitis is caused by a wide variety of Acanthamoeba spp., most of which belonging to the T4 rDNA complex [3]. The high proportion of T4 Acanthamoeba associated with keratitis could be attributed to its greater virulence and wide spreading in the environment with respect to other genotypes [8]. At present, also the T2, T3, T5, T6, and T11 genotypes have been associated with AK [3, 8-12]. The other genotypes have been found related to non-AK infections and/or environmental sources [13]. 
In Italy the first report of Acanthamoeba human infection dates back to the 1990s in one HIV-positive patient with meningoencephalitis [14], followed by a clinical case of one patient with bilateral keratitis $[15,16]$. A few years later three cases of Acanthamoeba keratitis associated with the use of contact lenses were reported by Mancino et al. [17]. Evidence of Acanthamoeba infection rates in Italy comes indirectly from serological investigations, where $3.7-4.5 \%$ of people showed antibodies against Acanthamoeba castellanii [18]. As regards the occurrence of Acanthamoeba in the environment, only one survey has hitherto been carried out in Italy, reporting a percentage of $5.2 \%$ of Acanthamoeba spp. in samples isolated from water and mud, collected in thermal baths and mud-basins [19].

Considering the very limited data concerning Acanthamoeba associated AK from Italy and the absence of information on genotype distribution in clinical samples, the aim of this work was to evaluate the occurrence of Acanthamoeba in patients with ocular infections, to characterize the recovered isolates at molecular level by sequencing a portion of the 18S rRNA gene (Rns), and to analyse the degree of genetic variation at intra-genotype level by sequencing the DF3 highly variable region.

\section{Materials and methods}

From January 2006 to February 2008, a total of 140 patients (65 males and 75 females), admitted to the Ophthalmology Clinic (Policlinico Hospital, University of Bari) for ocular infections, were examined for Acanthamoeba. One hundred twenty-three corneal swabs or corneal scrapings, 26 conjunctival swabs, one vitreous specimen, and 57 contact lens or lens case solutions were analysed. Both conjunctival/ corneal swabs or corneal scraping and contact lenses or lens case solutions were obtained from 53 patients.

\section{Amoeba culture}

Amoeba culture was performed according to Khan [7]. Briefly, clinical specimens were transferred to Page's amoeba saline and centrifuged at $800 \times \mathrm{g}$ for $5 \mathrm{~min}$. The sediment was suspended. Isolation of Acanthamoeba was performed on $1.5 \%$ non-nutrient agar (Difco, Milan, Italy) made with Page's amoeba saline and overlayed with a 24-h old culture of Escherichia coli. The precoated agar plates were incubated with 2-3 drops of the above suspension and incubated at $30^{\circ} \mathrm{C}$. The plates were examined for trophozoites and cysts directly under microscope daily for 7 days. When amoebae were detected, a small piece of agar was cultured on the surface of a fresh agar plate pre-coated with bacteria and incubated as described above to maintain the strains. Plates were stored at $4^{\circ} \mathrm{C}$ for the molecular studies.
Molecular procedures

The growing amoebae were harvested from culture plates with a sterile applicator, placed in Eppendorf tubes with $100 \mu 1$ of physiological solution, and mixed by a vortex. DNA extractions were performed using the QIAamp DNA Micro Kit (Qiagen, Italy). The PCR procedure was carried out to amplify a 405-bp region from the 18S-rRNA gene (Rns) defined as ASA.S1 that includes Diagnostic Fragment 3 (DF3), using the genus-specific primers JDP1 and JDP2 [13]. The ASA.S1 fragment allows the specific detection of Acanthamoeba since it is discriminating for the genus and can be obtained from all known 18S rDNA genotypes [13]. The amplifications were carried out in a $25 \mu \mathrm{l}$ volume containing $12.5 \mu \mathrm{l}$ PCR master mix $2 \mathrm{X}$ (Promega, Italy), $5 \mu$ template DNA, and $0.6 \mathrm{mM}$ of each primer and were performed in a iCycler Thermal Cycler (BioRad, Italy).

The cycling conditions were: a denaturing step at $96^{\circ} \mathrm{C}$ for $2 \mathrm{~min}, 35$ cycles of denaturing for $1 \mathrm{~min}$ at $96^{\circ} \mathrm{C}$, annealing for $1 \mathrm{~min}$ at $60^{\circ} \mathrm{C}$, and extension for $1 \mathrm{~min}$ at $72^{\circ} \mathrm{C}$, followed by a final extension for $7 \mathrm{~min}$ at $72^{\circ} \mathrm{C}$. One negative control was included in each batch of DNA extraction and PCR reaction. The PCR products were visualised by electrophoresis on $1 \%$ agarose gel stained by SYBR Safe DNA gel stain (Invitrogen). PCR products with the expected size were excised from the gel and DNA was purified using a NucleoSpin Extract (MachereyNagel) purification kit and sequenced for both strands by Bio-Fab Research (Italy).

\section{Sequence analysis}

Assignment to genotypes was performed by sequence comparison and phenetic analysis.

Multiple alignments were performed using ClustalW2 [20] in comparison with sequences available in GenBankTM for Acanthamoeba genotypes under the following accession numbers: T1: A. castellanii CDC:0981:V006 (U07400); T2: A. palestinensis Reich; ATCC 30870 (AF479563); T3: A. griffini S-7 ATCC 30731 (U07412); T4: A. castellanii Neff ATCC 50373 (U07416); T4: Acanthamoeba sp. S27 (DQ087323); T5: A. lenticulata PD2S (U94741); T6: $A$. palestinensis 2802 (AF019063); T7: A. astronyxis CCAP 1534/1 (AF239293); T8: A. tubiashi OC-15C (AF019065); T9: A. comandoni Comandon \& de Fonbrune (AF019066); T10: Acanthamoeba sp. CDC \# V369 (AY703001); T11: Acanthamoeba sp. PN14 (AF333608); T12: A. healyi CDC 1283:V013 (AF019070); T13: Acanthamoeba sp. UWC9 (AF132134); T14: Acanthamoeba sp. PN15 (AF333607); and T15: A. jacobsi AC305 (AY262365).

The 14 sequences obtained in our study were deposited in the GenBankTM database and are available under the 
accession numbers: EF654665-EF654667, EU741250EU741257, and FJ195367-FJ195369 (Table 1).

The phenetic relationships within the genus were studied based on both the ASA.S1 and DF3 sequences. Three distinct datasets were analysed, one including the entire sequence, one excluding the DF3 region, and one represented only by the DF3. The first two data sets were used for distance-based analyses performed by MEGA version 4 [21], conducted using the maximum composite likelihood distance estimates, and trees were constructed by the Neighbour-Joining algorithm. Bootstrap values were calculated by analysing 1,000 replicates. The intra-genotype genetic variation was assessed by comparison of the highly variable region DF3.

\section{Results}

Nineteen out of 140 (13.6\%) patients had Acanthamoeba culture positive samples, of which 18 were contact lens wearers. In nine patients Acanthamoeba was isolated from ocular specimens only, in four patients from both corneal swabs and contact lenses, and in six cases only from contact lenses. Clinical presentations ranged from chronic conjunctivitis to corneal abscess or ulceration, keratitis, and panuveitis in one patient.

In particular, one case of chronic conjunctivitis was described in a no lens wearer patient. In this case Acanthamoeba was isolated from a conjunctival swab in October 2006, but for the unusual clinical picture, no specific treatment for Acanthamoeba was undertaken. Considering the persistence of conjunctivitis-related symptoms, another conjunctival swab was examined 6 months later-again, Acanthamoeba sp. was isolated and a specific therapy was started with complete resolution of conjunctivitis. Another patient displayed a severe bilateral keratitis.
The same strain of Acanthamoeba was isolated from corneal swabs of both eyes and from both contact lenses.

As for the molecular characterization of the isolates, interpretable sequences were obtained from 14 out of 19 positive samples (Table 1).

Eleven out of the 14 sequences revealed a strict correspondence with the available sequences of the T4 genotypes. The percentage of identity between the obtained sequences and the two T4 sequences chosen as reference (accession numbers DQ087323 and U07416) ranged from $96.81 \%$ to $100 \%$ using the entire dataset and from $98.69 \%$ to $100 \%$ using the dataset excluding the highly variable region DF3. The two NJ trees obtained using the datasets including and excluding the DF3 variable region revealed similar topologies (Figs. 1 and 2, respectively). The trees showed that 11 isolates are strictly related with each other and with two Acanthamoeba castellanii genotype T4 chosen as references. Three of the 14 isolates analysed (ACA5, ACA12, and ACA14) showed a strict correspondence with the deposited sequences for the genotype T15, corresponding to the species A. jacobsi, with $100 \%$ of sequence identity with the T15 sequence chosen as reference (accession number AY262365) using both the "entire" and the "excluding DF3" datasets.

All of the above correspondence were strongly supported, as depicted by the high bootstrap values obtained in genotype assignments. On the other hand, the overall tree topology is not always supported by significant bootstrap values.

The clinical pictures of the three patients with genotype T15 Acanthamoeba infection were as follows. The first patient, a contact lens wearer, displayed symptoms that included pain, tearing, conjunctivitis, photophobia, and decreased vision in the right eye for about 1 month, unresponsive to empirical antibiotic therapy. On examination, the right eye showed a ring-shaped corneal infiltrate

Table 1 Origin, clinical data, accession numbers and genotype of the Acanthamoeba isolates under study

\begin{tabular}{llllll}
\hline Isolate & Source & Clinical presentation & Contact lens wearer & GenBankTM Accession Number & Genotype \\
\hline Aca1 & Conjunctival swab & Chronic conjunctivitis & No & EF654665 & T4 \\
Aca2 & Corneal scraping & Corneal ulceration & Yes & EF654666 & T4 \\
Aca3 & Corneal swab & Corneal ulceration & Yes & EF654667 & T4 \\
Aca4 & Corneal swab & Corneal abscess & Yes & EU741250 & T4 \\
Aca5 & Corneal swab & Corneal abscess & Yes & EU741252 & T15 \\
Aca6 & Corneal swab & Multiple corneal abscess & Yes & EU741253 & T4 \\
Aca7 & Corneal swab & Corneal abscess & Yes & EU741254 & T4 \\
Aca8 & Corneal scraping & Keratitis & Yes & EU741255 & EU741256 \\
Aca9 & Corneal swab & Bilateral keratitis & Yes & EU741257 & T4 \\
Aca10 & Corneal swab & Keratitis & Yes & FJ195367 & T4 \\
Aca11 & Corneal swab & Corneal abscess & Yes & FJ195368 & T4 \\
Aca12 & Corneal swab & Multiple corneal abscess & Yes & Yes & Tes \\
Aca13 & Contact lens & Corneal abscess & Panuveitis & T4 & T15 \\
Aca14 & Vitreous specimen & & &
\end{tabular}




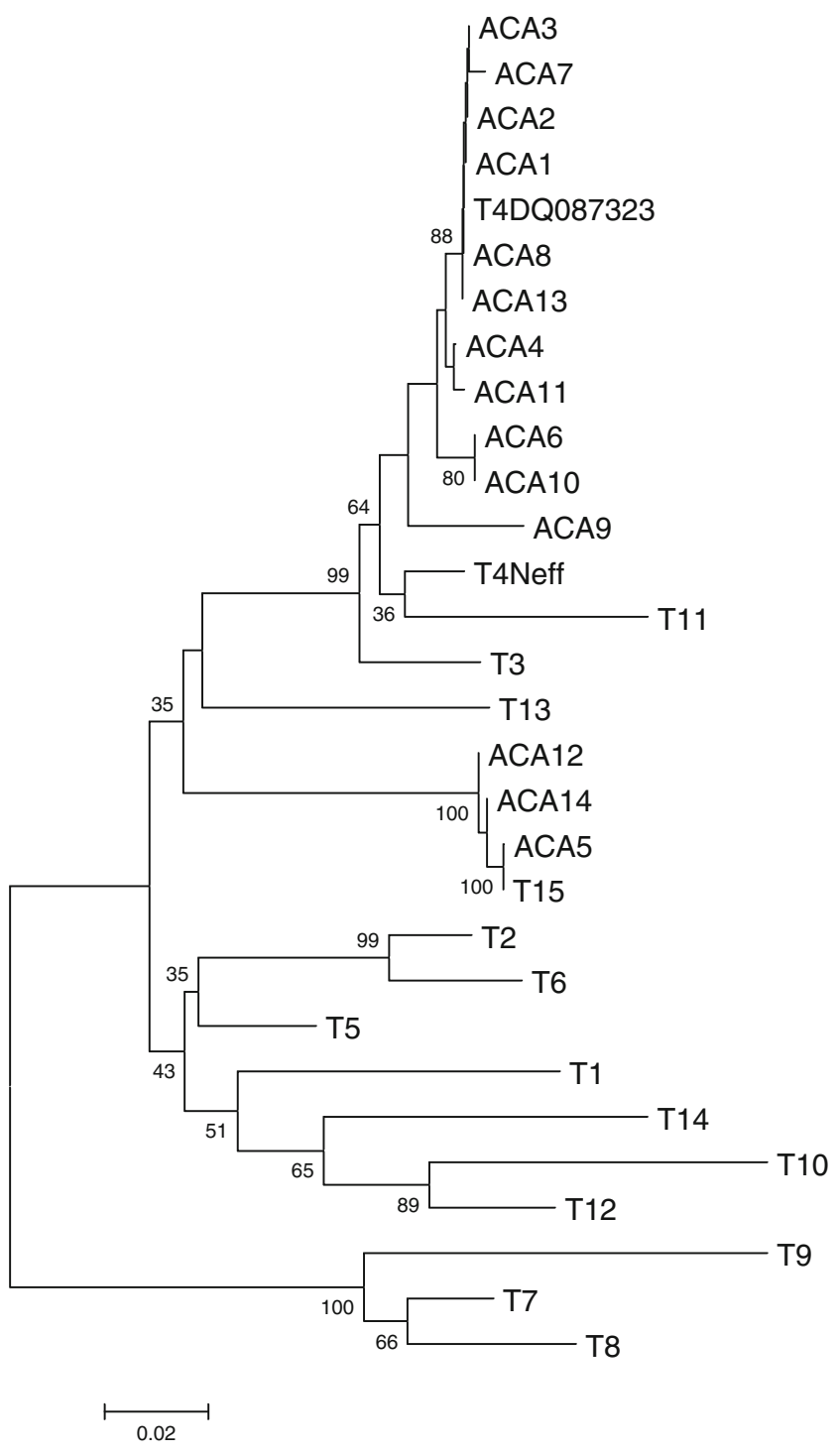

Fig. 1 18S rDNA linearized neighbour-joining tree based on the entire dataset (including DF3) obtained by using the maximum composite likelihood distance estimates, 1000 bootstrap replications, and produced in MEGA 4.0. Abbreviations for Italian isolates are as defined in Table 1 . The T1-T15 designations correspond to sequences previously determined to be of that particular genotype and available in GenBankTM under the accession numbers as reported in Materials and Methods

with diffuse stromal haze. Ipopion was also present. Culture of a corneal swab revealed the growth of Acanthamoeba and absence of bacteria and fungi. The patient was treated with topical $0.02 \%$ Polyhexamethylene biguanide (PHMB) plus $0.02 \%$ chlorexidine instilled every 4 hours/day for 3 months plus neomycin. The patient also received oral fluconazole. A reduction of inflammation, re-epithelialization, and a partial restoration of visual acuity were obtained. The second patient, a contact lens wearer, presented with pain, redness, photophobia, and burned vision in the right eye of 20 days duration. A diagnosis of keratitis was made for the presence of multiple corneal infiltrates. He was previously empirically treated with gancyclovir because an herpetic keratitis was suspected. The corneal swab was culture positive for Acanthamoeba but not bacteria and fungi. The patient was topically treated with chlorexidine $0.02 \%$ plus propamidine isethionate $0.1 \%$ (Brolene) instilled every 4 hours/ day for 3 months plus neomycin. Oral fluconazole was also administered. Reduction of inflammation and a partial restoration of visual acuity were observed. The third patient, a former drug-user and HIV-negative, was admitted with a

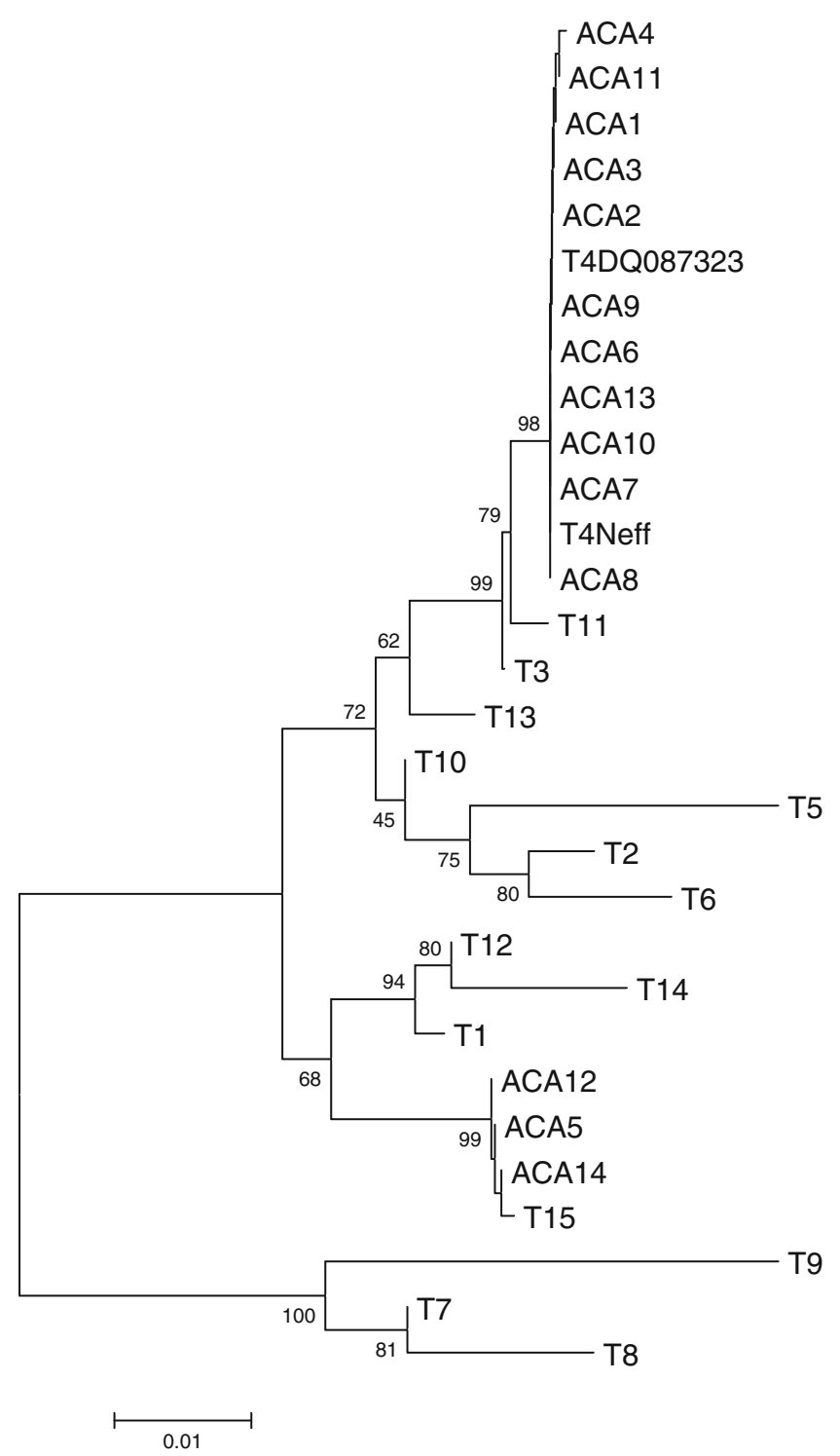

Fig. 2 18S rDNA linearized neighbour-joining tree based on the "excluding df3" dataset obtained by using the maximum composite likelihood distance estimates, 1000 bootstrap replications, and produced in MEGA 4.0. Abbreviations for Italian isolates are as defined in Table 1. The T1-T15 designations correspond to sequences previously determined to be of that particular genotype and available in GenBankTM under the accession numbers as reported in Materials and Methods 
clinical diagnosis of panuveitis. The patient also presented granulomatous skin lesions that were not microbiologically investigated. Acanthamoeba was isolated from one vitreous specimen. The sample was negative for bacteria and fungi and PCR positive for Epstein Barr virus. Unfortunately, no further data are available on the development of clinical signs.

The analysis of the DF3 variable region (alignment reported in Fig. 3) revealed a percentage of variation among the isolates identified as belonging to the $\mathrm{T} 4$ genotype ranging from $0 \%$ to $15 \%$. Conversely, no nucleotide variation was observed between the ACA5, ACA12, and ACA14 isolates, identified as T15 and the reference sequence AY262365. Interestingly, the analysis of the DF3 region also showed an identity of $100 \%$ when comparing the DF3 sequences of the six T15 genotype isolates so far available in GenBankTM.

\section{Discussion}

The classification of the genus Acanthamoeba is still under debate and the precise identification of strains requires molecular approaches. At least 24 named species have been described within this genus, many of them containing both pathogenic and non pathogenic isolates [11, 22]. Pussard and Pons [23] classified the genus into three morphological groups; however, this classification has proven to be unreliable due to alternating cyst appearance. The analysis of the 18S rDNA proved to be an effective tool for describing dissimilarities in the genus, thus identifying 12 distinct sequence types (T1-T12) [3, 9]. Three more sequence types, T13 [4], T14 [5] and T15 [6], so far not related to a precise pathogenic picture, have been further described.

This study represents the first characterization of Acanthamoeba genotypes in Italy, in isolates obtained from patients showing keratitis-related symptoms.

Eleven out of the 14 isolates genetically characterized were assigned to the $\mathrm{T} 4$ genotype. The high proportion of Rns genotype T4 strains has been described as the main factor responsible for Acanthamoeba keratitis infections in many countries, i.e. in France (8/11, plus additional recent data) [24, 25], in Greece (4/5) [12], in Hong Kong (12/13) [26], and in the UK and Iran (17/24) [8], thus providing further support to the evidence that the T4 genotype plays a prominent role in the epidemiology of AK human infections.

Consistent genotype assignments were obtained using two datasets based on sequence alignment of the $18 \mathrm{~S}$ nuclear ribosomal DNA, one based on the entire dataset and the other excluding the DF3 region. The DF3 is one out of three highly variable portions of the $18 \mathrm{~S}$ nuclear ribosomal DNA, and phylogenetic trees based on these regions are considered useful to better analyse the genetic variability among the isolates.

However, the topology of the two trees, as evidenced in Figs. 1 and 2 (including and excluding DF3), seems to indicate that the DF3 region is a good target to detect intragenotype variation, at least for the T4 genotype, rather than as a tool for genotype assignment. In fact, if excluding the DF3 region, higher bootstrap values allow a better resolution of the relationships between the closely related members of the T3-T4-T11 cluster [13, 22].

Three isolates were identified as belonging to the T15 genotype, putatively assignable to the species Acanthamoeba jacobsi. This finding represents the first record of human keratitis due to the T15 genotype, as confirmed by clinical criteria at least in two out of three cases, thus likely extending the range of Acanthamoeba genotypes able to determine such pathogenic effect in humans. This is noteworthy, since genotype T15 includes only six isolates assigned to the species $A$. jacobsi, isolated from different environmental sources with a wide geographic distribution [6].

Infections caused by pathogenic free-living Acanthamoeba represent an emerging disease in humans. Because of technical difficulties in diagnosis, these infections are likely to be under-diagnosed. The amplification with primers JDP1 and JDP2 proved to be a sensitive method, potentially able to detect Acanthamoeba without the need for long culture incubation, and thus considerably useful for clinical applications.
Fig. 3 Sequence alignment of variable region of DF3. The alignment shown is a subset of DF3 that contains the highly variable and informative section of this fragment. Abbreviations for samples and relative genotype assignments are as defined in Table 1

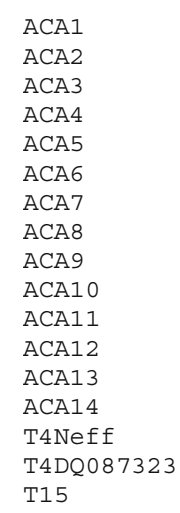

GGTGCGGTCG TCCTTGGCGT CTCGGTCCTT CACGGGGCCG GGGCGCGGGG GCGGC-T-AG CCCGG

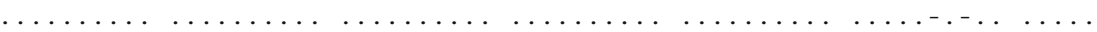

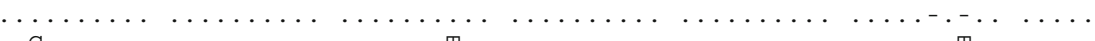
.С... ‥ . .GT... . ....... TG TCGT.CG.GG .GAC.C..GC .CG..A. ...TG.CT. ...

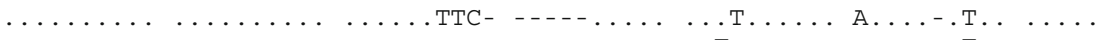

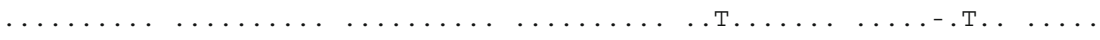

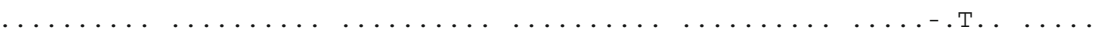

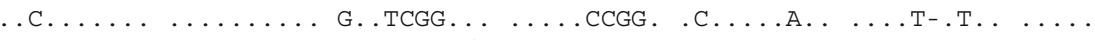

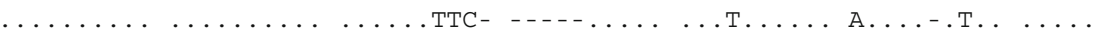
.С... ......

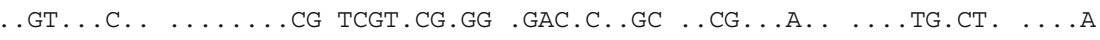

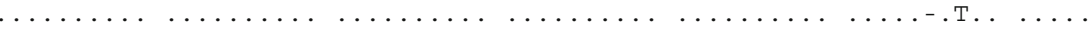

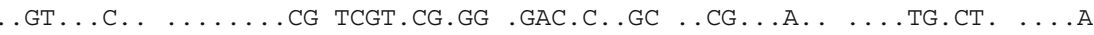

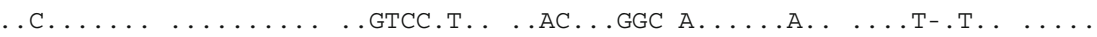

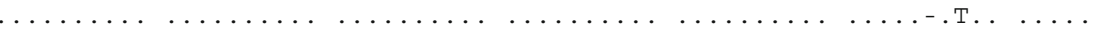
.GT..C. ....... 


\section{References}

1. Marciano-Cabral F, Puffenbarger R, Cabral GA (2000) The increasing importance of Acanthamoeba infections. J Eukaryot Microbiol 47:29-36. doi:10.1111/j.1550-7408.2000.tb00007.x

2. Page FC (1988) A new key to freshwater and soil gymnamoebae. Freshwater Biological Association, Ambleside, UK

3. Stothard DR, Schroeder-Diedrich JM, Awwad MH, Gast RJ, Ledee DR, Rodriguez-Zaragoza S, Dean CL, Fuerst PA, Byers TJ (1998) The evolutionary history of the genus Acanthamoeba and the identification of eight new $18 \mathrm{~S}$ rRNA gene sequence types. J Eukaryot Microbiol 45:45-54. doi:10.1111/j.1550-7408.1998. tb05068.x

4. Horn M, Fritsche TR, Gautom RK, Schleifer KH, Wagner M (1999) Novel bacterial endosymbionts of Acanthamoeba spp. related to the Paramecium caudatum symbiont Caedibacter caryphilus. Environ Microbiol 1:357-367. doi:10.1046/j.14622920.1999.00045.x

5. Gast RJ (2001) Development of an Acanthamoeba specific reverse dot-blot and the discovery of a new ribotype. J Eukaryot Microbiol 48:609-615. doi:10.1111/j.1550-7408.2001.tb00199.x

6. Hewett MK, Robinson BS, Monis PT, Saint CP (2003) Identification of a new Acanthamoeba 18S rRNA gene sequence type corresponding to the species Acanthamoeba jacobsi Sawyer, Nerad and Visvesvara, 1992 (Lobosea: Acanthamoebidae). Acta Protozool 42:325-329

7. Khan NA (2006) Acanthamoeba: biology and increasing importance in human health. FEMS Microbiol Rev 30:564-595. doi:10.1111/j.1574-6976.2006.00023.x

8. Maghsood AH, Sissons J, Rezaian M, Nolder D, Warhurst D, Khan NA (2005) Acanthamoeba genotype T4 from the UK and Iran and isolation of the T2 genotype from clinical isolates. J Med Microbiol 54:755-759. doi:10.1099/jmm.0.45970-0

9. Gast RJ, Ledee DR, Fuerst PA, Byers TJ (1996) Subgenus systematics of Acanthamoeba: four nuclear 18S rDNA sequence types. J Eukaryot Microbiol 43:498-504. doi:10.1111/j.15507408.1996.tb04510.x

10. Walochnik J, Haller-Schober E-M, Kölli H, Picher O, Obwaller A, Aspöck H (2000) Discrimination between clinically relevant and nonrelevant Acanthamoeba strains isolated from contact lenswearing keratitis patients in Austria. J Clin Microbiol 38:3932-3936

11. Khan NA, Jarroll EL, Paget TA (2002) Molecular and physiological differentiation between pathogenic and non-pathogenic Acanthamoeba. Curr Microbiol 45:197-202. doi:10.1007/ s00284-001-0108-3

12. Spanakos G, Tzanetou K, Miltsakakis D, Patsoula E, MalamouLada E, Vakalis NC (2006) Genotyping of pathogenic Acanthamoebae isolated from clinical samples in Greece-report of a clinical isolate presenting T5 genotype. Parasitol Int 55:147-149. doi:10.1016/j.parint.2005.12.001

13. Schroeder JM, Booton GC, Hay J, Niszl IA, Seal DV, Markus MB et al (2001) Use of subgenic 18S ribosomal DNA PCR and sequencing for genus and genotype identification of Acantha- moebae from humans with keratitis and from sewage sludge. J Clin Microbiol 39:1903-1911. doi:10.1128/JCM.39.5.19031911.2001

14. Di Gregorio C, Rivasi F, Mongiardo N, De Rienzo B, Wallace S, Visvesvara GS (1992) Acanthamoeba meningoencephalitis in a patient with acquired immunodeficiency syndrome. Arch Pathol Lab Med 116:1363-1365

15. Manso E, Biavasco F, Lupidi R, Giovannini A, Frongia G, Varaldo PE (1993) Primo isolamento in Italia di un ceppo di Acanthamoeba da un paziente affetto da cheratite bilaterale. Microbiol Med 8:100-104

16. Giovannini A, Tittarelli R, Bertelli E, Frongia GB, Mariotti C, Manso E, Biavasco F (1994) Bilateral Acanthamoeba keratitis in a gas-permeable contact lens wearer. Ophthalmologica 208:321324

17. Mancino R, Iori A, Palma S, Corsi A, Cancrini G, Cerulli L (1997) Acanthamoeba keratitis associated with contact lenses; report of three cases in Italy. Parassitologia 39:37-40

18. Pennisi L, Mento G, Todaro F (1971) Sulla diffusione di anticorpi anti-Acanthamoeba castellanii in soggetti provenienti da varie regioni italiane. Parassitologia 13:299-308

19. Scaglia M, Gatti S, Brustia R, Strosselli M, Bernuzzi AM, Cevini C (1987) Pathogenic and non-pathogenic Naegleria and Acanthamoeba spp.: a new autochthonous isolate from an Italian thermal area. Microbiologica 10:171-182

20. Larkin MA, Blackshields G, Brown NP, Chenna R, McGettigan PA, McWilliam H et al (2007) ClustalW and ClustalX version 2. Bioinformatics 23:2947-2948. doi:10.1093/bioinformatics/ btm404

21. Tamura K, Dudley J, Nei M, Kumar S (2007) MEGA4: molecular evolutionary genetics analysis (MEGA) software version 4.0. Mol Biol Evol 24:1596-1599. doi:10.1093/molbev/msm092

22. Booton GC, Visvesvara GS, Byers TJ, Kelly DJ, Fuerst PA (2005) Identification and distribution of Acanthamoeba species genotypes associated with nonkeratitis infections. J Clin Microbiol 43:1689-1693. doi:10.1128/JCM.43.4.1689-1693.2005

23. Pussard M, Pons R (1977) Morphologies de la paroi kystique et taxonomie du genre Acanthamoeba (Protozoa, Amoebida). Protistologica 13:557-598

24. Yera H, Zamfir O, Bourcier T, Ancelle T, Batellier L, DupouyCamet J, Chaumeil C (2007) Comparison of PCR, microscopic examination and culture for the early diagnosis and characterization of Acanthamoeba isolates from ocular infections. Eur J Clin Microbiol Infect Dis 26:221-224. doi:10.1007/s10096-007-0268-6

25. Yera H, Zamfir O, Bourcier T, Viscogliosi E, Noël C, DupouyCamet J, Chaumeil C (2008) The genotypic characterization of Acanthamoeba isolates from human ocular samples. Br J Ophthalmol 92:119-1141. doi:10.1136/bjo.2007.132266

26. Booton GC, Kelly DJ, Chu YW, Seal DV, Houang E, Lam DS, Byers TJ, Fuerst PA (2002) 18S ribosomal DNA typing and tracking of Acanthamoeba species isolates from corneal scrape specimens, contact lenses, lens cases, and home water supplies of Acanthamoeba keratitis patients in Hong Kong. J Clin Microbiol 40:1621-1625. doi:10.1128/JCM.40.5.1621-1625.2002 